\title{
Empirical Research on Systematic Evaluation of XX Precise Machining Company's Production Capacity
}

\author{
Tang Hong
}

Xi'an International University, Xi'an, Shaanxi, 710077

Keywords. structural modeling; level analysis; fuzzy evaluation

\begin{abstract}
By use of system engineering methods such as structural modeling, level analysis, fuzzy evaluation, this paper conducts analysis of main factors contributing to XX Precise Machining Company's production capacity to create a production capacity evaluation factor structural model and perform evaluation. Take XX Precise Machining Company as empirical research object, this paper analyses the key impact factors contributing to company's production capacity and sets up a evaluation factor structure model to evaluate its production capacity by using system engineering methods as structural modeling, level analysis, and fuzzy evaluation.
\end{abstract}

\section{Introduction}

XX Precise Machining Company under AVIC I, is mainly engaged in machining aircraft engine hydraulic device and conduction parts and has passed ISO9000 Quality System Certification, with robust strength. XX Precise Machining Company explores and strengthens the domestic market, but also open up overseas market at the same time to precisely machine parts of large foreign civil aircraft. The company's current production, technology and equipment conditions cannot satisfy foreign customers' high-precision and strict appearance requirements for some products. So, to obtain profit, the company must guarantee product quality and prompt delivery and get good reputation. Now that there are three types of foreign products (A, B, C), the company has to decide which satisfies customer requirements. For this reason, the company needs to evaluate the current production capacity and make decisions. XX Precise Machining Company is a subsidiary company of AVIC I. The principal products of the company are hydraulic devices of aircraft engine and conduction parts. It is a powerful company and its products have passed ISO9000 Quality System Certification. XX Precise Machining Company not only explores and strengthens the domestic market, but also develops the overseas market. The overseas market mainly focuses on precisely processing components and parts of large civil aircraft. The company's current production technology and equipment cannot satisfy foreign customers' strict and high-standard requirements on products' appearance and precision. In order to earn reputation and make profits, the company should guarantee the quality of the products and delivery date. Now there are three types of foreign products $\mathrm{A}, \mathrm{B}$, and $\mathrm{C}$, the company has to make one choice from them and then produce the selected one. The company must make sure that the selected production could meet the requirements of foreign customers. So the company needs to evaluate the current production capacity and make the decision. 
On an ad hoc basis of foreign orders (the company shall only satisfy for product design requirement and delivery considering machining on order from foreign customers), here main factors contributing to the company's production capacity are analyzed by system engineering methods such as structural modeling, level analysis, fuzzy evaluation. Based on the facts of foreign order (it is a processing trade, the company only needs to meet the requirements of product design and shipment), this paper analyses the key impact factors of company's production capacity by system engineering methods as structural modeling, level analysis and fuzzy evaluation.

\section{Establishment of Evaluation Index System}

Give a list of system factors a list of system factors

By gathering opinions method, opinions of the company leadership, technical staff including workshop chief, group leader and some staff from technical office and production office as well as experienced workers are collected to sum up 14 main factors contributing to the production capacity shown as system factors in Table 1-1: By gathering opinion methods, this paper induces 14 key impact factors of company's production capacity based on the opinions of leadership, technical staff including workshop chief, group leaders and some experienced workers from production lines. The system factors as shown in Table 1-1.

Table 1-1

Creation of reachable matrix Establishing reachable matrisx

By relevant staff's repeated analysis and discussion in each unit, pairwise relation between the above factors is shown as Fig. 1-1. Through repeated analyses and discussion, the staffs from different departments find out corresponding relationship between above factors.

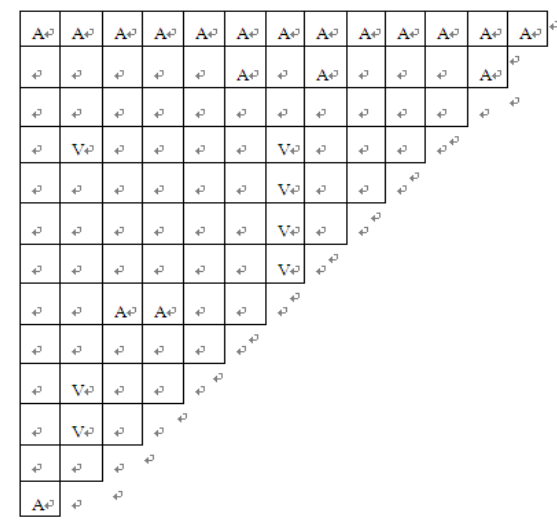

Fig. 1-1 One-to-One Relationship between the Factors

In Fig. 1-1, "A" means the lower factor is strongly correlated to the upper factor (the former reflects the latter well), "V" indicates the upper factor strongly correlated to the lower factor (the former reflects the latter well).

On this basis, reachable matrix $\mathrm{R}$ used to evaluate direct and indirect relations between the factors (“/”. shows two factors directly or indirectly correlated, and "empty" shows slightly correlated or uncorrelated, as shown in Fig. 1-2). Based on this relationship, reachable matrix could be used to evaluate direct and indirect relationship between the factors. 


\begin{tabular}{|l|l|l|l|l|l|l|l|l|l|l|l|l|l|}
\hline$/$ & & & & & & & & & & & & & \\
\hline$/$ & $/$ & & & & & & & & & & & & \\
\hline$/$ & $/$ & $/$ & & & & & & & & & & & \\
\hline$/$ & & & $/$ & & & & $/$ & & & & & $/$ & \\
\hline$/$ & & & & $/$ & & & $/$ & & & & & & \\
\hline$/$ & & & & & $/$ & & $/$ & & & & & & \\
\hline$/$ & $/$ & & & & & $/$ & $/$ & & & & & & \\
\hline$/$ & & & & & & & $/$ & & & & & & \\
\hline$/$ & $/$ & & & & & & & $/$ & & & & & \\
\hline$/$ & & & & & & & & & $/$ & & & $/$ & \\
\hline$/$ & & & & & & & $/$ & & & $/$ & & $/$ & \\
\hline$/$ & & & & & & & $/$ & & & & $/$ & & \\
\hline$/$ & & & & & & & & & & & & $/$ & \\
\hline$/$ & & & & & & & & & & & & $/$ & $/$ \\
\hline
\end{tabular}

Fig. 1-2 Reachable Matrix of Factors Evaluations

\section{Level classification}

It is aimed at classifying all evaluation factors to draw a clear-cut layered structure of the index framework (system). The aim of classifying is to divide evaluation factors into different levels and clear the hiberarchy of evaluation index system.

For each factor $\mathrm{Pi}$, define the set composed of its reachable factors as reachable set $\mathrm{R}(\mathrm{Pi})$ and the set composed of factors reaching $\mathrm{Pi}$ as the former factor set $\mathrm{A}(\mathrm{Pi})$. For each factor $\mathrm{Pi}$, the set of reachable factors is defined as reachable set $\mathrm{R}(\mathrm{Pi})$ and the set of factors reached $\mathrm{Pi}$ is defined as former factor set $\mathrm{A}(\mathrm{Pi})$.

Iterative algorithm for level classification is indicated as below:

$\mathrm{Lj}=\{$ Pì $\in$ P-L0- L1- $\cdots$ - Lj-1 $\mid$ Rj-1(Pì) $\cap$ Aj-1(Pì)= Rj-1(Pì) $\}$

In the equation, $\mathrm{L} 1, \mathrm{~L} 2 \cdots \mathrm{Ll}$ indicate all the levels from top to bottom, then level classification $\cap:(\mathrm{P})$ can be written as:

$\cap:(\mathrm{P})=(\mathrm{L} 1, \mathrm{~L} 2, \cdots, \mathrm{Ll}) \mathrm{l}$ is series.

Rj-1(Pì) and Aj-1(Pì) are respectively reachable set and former factor set obtained by calculating subfactors composed of factors in P-L0- L1- ... - Lj-1. See Tables 1-2 to 1-4 for the solution procedure of level classification.

Table 1-2

\begin{tabular}{|c|c|c|c|c|c|}
\hline \multicolumn{2}{|c|}{ Evaluation Factor Lip } & $\begin{array}{c}\text { Reachable Set } \\
\mathrm{R}(\mathrm{B} i)^{2}\end{array}$ & $\begin{array}{c}\text { Fomer Factor Set } \\
\text { A(pit) }\end{array}$ & $\mathrm{R} \cap \mathrm{A}$ & \multirow{2}{*}{$\begin{array}{l}\mathrm{R} \cap \mathrm{A}=\mathrm{R} \\
1\left(\mathrm{I}_{1}\right) \\
\end{array}$} \\
\hline & 1 & 1 & $1-14$ & 1 & \\
\hline & 20 & 1,2 & $2,3,7,9$ & 2 & 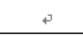 \\
\hline & 3 & $1,2,3$ & 30 & 3 & 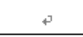 \\
\hline & 4 & $1,4,8,13$ & 4 & 4 & 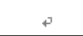 \\
\hline & 5 & $1,5,8 \mathrm{~B}$ & 5 & 5 & 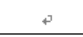 \\
\hline & 6 & $1,6,8$ & 6 & 6 & 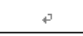 \\
\hline & 70 & $1,2,7,8$ & 7 & 70 & . \\
\hline P- $\mathrm{L}_{0}$ & 8 & 1,8 & $4,5,6,7,7,8,11,12$ & 8 & 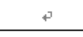 \\
\hline & 9 & $1,2,9$ & 9 & 9 & 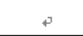 \\
\hline & 10 & $1,10,13$ & 10 & 10 & 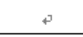 \\
\hline & 11 & $1,8,11,13$ & 11 & 11 & 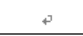 \\
\hline & 12 & $1,8,12$ & 12 & 12 & 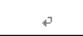 \\
\hline & 13 & 1,13 & $4,10,11,13,14$ & 13 & 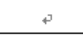 \\
\hline & $\mathrm{L}_{14}$ & $1,13,14$ & 14 & 14 & 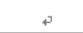 \\
\hline
\end{tabular}


Table 1-3

\begin{tabular}{|c|c|c|c|c|c|c|}
\hline \multicolumn{2}{|c|}{ Evaluation Factor Lip } & $\begin{array}{c}\text { Reachable Set } \\
\mathrm{R}(\mathrm{P} i)^{2}\end{array}$ & $\begin{array}{c}\text { Former Factor Set } \\
\mathrm{A}(\mathrm{Pi})\end{array}$ & $R \cap A$ & \multicolumn{2}{|c|}{$\mathrm{R} \cap \mathrm{A}=\mathrm{R}$} \\
\hline & {$[2$} & 2 & $2,3,7,9$ & 2 & \multicolumn{2}{|c|}{$2+7$} \\
\hline & 3 & 2,3 & 3 & 3 & & 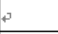 \\
\hline & 4 & $4,8,13$ & 4 & 4 & & 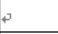 \\
\hline & 50 & 5,8 & 5 & 5 & & 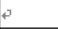 \\
\hline & 6 & 6,8 & 60 & 6 & & 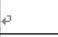 \\
\hline & 70 & $2,7,8$ & 7 & 7 & & 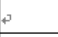 \\
\hline \multirow[t]{7}{*}{$\mathrm{P}-\mathrm{L}_{0}-\mathrm{L}_{1}$} & 8 & 80 & $4,5,6,7,8,11,12$ & 8 & 8 & $\mathrm{~L}_{2}$ \\
\hline & 9 & 2,9 & 9 & 9 & & 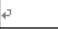 \\
\hline & 10 & 10,13 & 10 & 10 & & 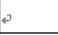 \\
\hline & 11 & $8,11,13$ & 11 & 11 & & 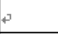 \\
\hline & 12 & 8,12 & 12 & 12 & & pe \\
\hline & 13 & $13+$ & $4,10,11,13,14$ & 13 & ] & \\
\hline & $\mathrm{L}_{14}$ & 13,14 & 14 & 14 & & 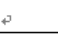 \\
\hline
\end{tabular}

Table 1-4

\begin{tabular}{|c|c|c|c|c|c|c|}
\hline \multicolumn{2}{|c|}{ Evaluation Factor Li } & $\begin{array}{c}\text { Reachable Set } \\
\text { R(Pi })\end{array}$ & $\begin{array}{c}\text { Former Factor Set } \\
\mathrm{A}(\mathrm{Pi})\end{array}$ & $\mathrm{R} \cap \mathrm{A}$ & \multicolumn{2}{|c|}{$\mathrm{R} \cap \mathrm{A}=\mathrm{R}$} \\
\hline & {$[3$} & 3 & 3 & 3 & 307 & \\
\hline & 4 & 4 & 4 & 4 & 40 & \\
\hline & 5 & 50 & 50 & 50 & 50 & \\
\hline & 6 & 6 & 6 & 6 & 6 & \\
\hline$P-L_{0}-L_{1}-L_{2}$ & 7 & 7 & 7 & 7 & 7 & $\mathrm{~L}_{3}$ \\
\hline & 9 & 9 & 9 & 9 & 9 & \\
\hline & 10 & 10 & 10 & 10 & 10 & \\
\hline & 11 & 11 & 11 & 11 & 11 & \\
\hline & 12 & 12 & 12 & 12 & 12 & \\
\hline & 14 & 14 & 14 & 14 & 14 & \\
\hline
\end{tabular}

The result is: $\cap:(\mathrm{P})=(1 ; 2,8,13 ; 3,4,5,6,7,9,10,11,12,14)$

Evaluation factor structure modeling

(1): By level classification $\cap:(P)$, obtain reachable matrix in level ranking, shown as Fig. 1-3:

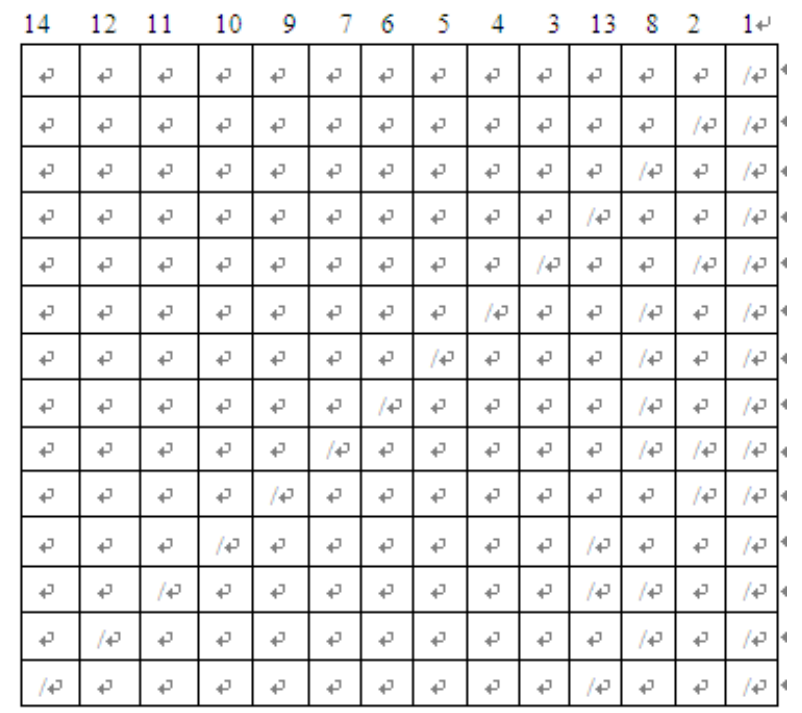

Fig. 1-3 Reachable Matrix in Level Ranking

(2): Building and interpretation of structure model Building and interpreting structure model

According to this, the structure model corresponding to reachable matrix is shown as Fig. 1-4. Take the second order factor as the first order evaluation index (mark it as B); the third factor as the second order evaluation index...There are 3 first-level indexes and 10 second-level indexes in the evaluation index structure. 


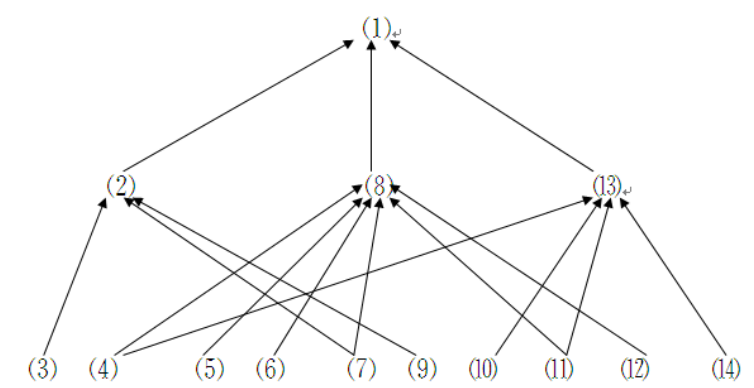

Fig. 1-4 Structure Model

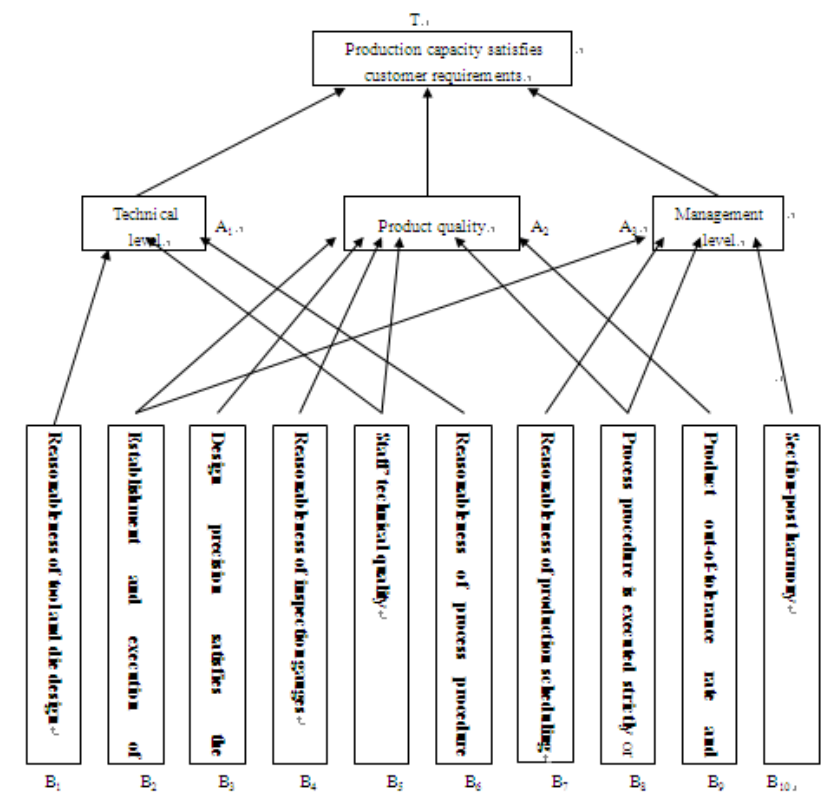

Fig. 1-5 Interpretation of Structure Model

System Evaluation

After initial definition of evaluation index system, various indexes shall not be treated equally to fulfill system evaluation, but give weights depending on their contributions to superior index and overall objective to establish a complete system evaluation index system. After the founding of initial evaluation index system,

Production capacity shown on Fig. 1-5 reaches the system evaluation index system of customer requirements to form a three-level (overall objective--first-level index--second-level index) analytic structure model so that importance weight of each index to superior index and overall objective can be determined by Analytic Hierarchy Process (AHP).

(1): Create evaluation matrix to determine priority weight of each factor.

By pairwise comparison of all the factors, determine their priority weights., with the numeric scale representing the relative importance of factor SI to factor Sj shown as Table 1-5:

Table 1-5

\begin{tabular}{|c|c|c|c|}
\hline $\begin{array}{c}\text { Criteria } \\
\text { Scale }\end{array}$ & Definition & $\begin{array}{c}\text { Criteria } \\
\text { Scale }\end{array}$ & Definition \\
\hline 1 & $\begin{array}{c}\text { Relative to } \mathrm{He}, \mathrm{S}_{\mathrm{I}} \text { and } \mathrm{S}_{\mathrm{j}} \text { are equally } \\
\text { important }\end{array}$ & 7 & $\begin{array}{c}\text { Relative to } \mathrm{He}, \mathrm{S}_{\mathrm{I}} \text { and } \mathrm{S}_{\mathrm{i}} \text { are much more } \\
\text { important }\end{array}$ \\
\hline 2 & $\begin{array}{c}\text { Relative to } \mathrm{He}, \mathrm{S}_{\mathrm{I}} \text { is slightly important } \\
\text { than } \mathrm{S}_{\mathrm{s}}\end{array}$ & 9 & $\begin{array}{c}\text { Relative to } \mathrm{He}, \mathrm{S}_{\mathrm{I}} \text { and } \mathrm{S}_{\mathrm{j}} \text { are absolutely } \\
\text { important }\end{array}$ \\
\hline 3 & $\begin{array}{c}\text { Relative to } \mathrm{He}, \mathrm{S}_{\mathrm{I}} \text { is more important } \\
\text { than } \mathrm{S}_{\mathrm{s}}\end{array}$ & $2,4,6,8$ & $\begin{array}{c}\text { Between the above neighbour criteria } \\
\text { scales }\end{array}$ \\
\hline
\end{tabular}

If $\mathrm{SI}$ is more important than $\mathrm{Sj}$, then $\mathrm{SIj}=5$; reversely, $\mathrm{SjI}=1 / 5$. 
Judge matrix A is the basic information of AHP and the basis for AHP. After normalization, the solution (W) to A's latent root $\mathrm{AW}=\lambda \operatorname{maxW}$ is the ordering weight of the same level to the superior level factor.

Set $\mathrm{A}=(\mathrm{aI}, \mathrm{j}) \quad \mathrm{I}, \mathrm{j}=1 \sim \mathrm{n}$

When conducting approximate solution by square root method, component of eigen vector is:

$\mathrm{WI}=(\Pi \mathrm{aI}, \mathrm{j}) 1 / \mathrm{n} \quad \mathrm{I}=1 \sim \mathrm{n}$

Max latent root $\lambda \max =\max \left(\sum(\mathrm{AW}) \mathrm{I} / \mathrm{WI}\right)$

Because of diversity in system property and human recognition's fuzziness, it is usually difficult to the created criteria matrix is completely consistent. However, AHP can be used for value analysis only if it has satisfactory consistency. So, consistency index (CI) shall satisfy the following conditions:

$$
\mathrm{CI}=(\lambda \max -\mathrm{n}) /(\mathrm{n}-1) \leqslant 0.10
$$

By expert investigation on and result analysis of all related personnel of the company' $\mathrm{s}$ various units, single ordering weight value of hierarchy (W 0 ) of the first-level index layer (A) can be obtained, with criteria matrix and process and result shown as List 1-6:

Table 1-6

\begin{tabular}{|c|c|c|c|c|c|c|c|}
\hline \multicolumn{4}{|c|}{$\begin{array}{l}\text { Expert I's criteria matrix A } \\
=(A+1)\end{array}$} & \multicolumn{2}{|c|}{ A eigen vector W } & \multicolumn{2}{|c|}{ A consistency check } \\
\hline $\mathrm{T}$ & $A_{1}$ & $\mathrm{~A}_{2}$ & $\mathrm{~A}_{3}{ }^{2}$ & $\mathrm{~W}_{\mathrm{r}}$ & $\mathrm{w}_{\mathrm{I}}{ }^{0}$ & $\lambda$ & $\mathrm{CI}$ \\
\hline $\mathrm{A}_{1}$ & 1 & $1 / 4$ & 3 & 0.909 & 0.226 & 3.085 & \\
\hline 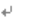 & 4 & & & + & & & \\
\hline $\mathrm{A}_{2}$ & 4 & 1 & 54 & 2.714 & 0.673 & 3.094 & $0.047 \leq 0.1$ \\
\hline$\checkmark$ & + & & & + & + & & \\
\hline $\mathrm{A}_{3}$ & $1 / 3$ & $1 / 5$ & 1 & 0.405 & 0.101 & 3.078 & \\
\hline
\end{tabular}

By the same principle, ordering weight values of hierarchy of the second order index layer (B) relative to first order index (A) shown as Tables 1-7, 1-8, 1-9 are obtained. Determination of importance weight of criteria index provides necessary information of the following comprehensive evaluation.

Table 1-7

\begin{tabular}{|c|c|c|c|c|c|c|c|}
\hline \multicolumn{4}{|c|}{$\begin{array}{l}\text { Expert I's criteria matrix A } \\
=\left(3 \mathrm{I}_{\mathrm{j}}\right)\end{array}$} & \multicolumn{2}{|c|}{ A eigen vector W } & \multicolumn{2}{|c|}{ A consistency check } \\
\hline $\mathrm{A}_{1}$ & $B_{1}$ & $B_{5}$ & $\mathrm{~B}_{6}$ & $\mathrm{~W}_{\mathrm{I}}$ & $\mathrm{W}_{\mathrm{I}}^{0} \mathrm{P}$ & $\lambda$ & $\mathrm{CI}$ \\
\hline$B_{1}$ & 1 & $1 / 5$ & $1 / 3$ & 0.405 & 0.109 & 3.013 & \\
\hline $\mathrm{B}_{5}$ & 5 & 1 & 24 & 2.154 & 0.582 & 2.998 & $0.0065 \leq 0.1$ \\
\hline $\mathrm{B}_{6}$ & 3 & $1 / 2$ & 1 & 1.145 & 0.309 & 3.000 & \\
\hline
\end{tabular}

Table 1-8

\begin{tabular}{|c|c|c|c|c|c|c|c|c|c|c|}
\hline \multicolumn{7}{|c|}{ Expert $I$ 's criteria matrix $\mathrm{A}=(\mathrm{a} \mathrm{i} ;)^{2}$} & \multicolumn{2}{|c|}{ A eigen vector $W$} & \multicolumn{2}{|c|}{ A consistency check } \\
\hline $\mathrm{A}_{2}$ & $\mathrm{~B}_{2}$ & $\mathrm{~B}_{3}$ & $\mathrm{~B}_{4}$ & $\mathrm{~B}_{5}$ & $\mathrm{~B}_{8}$ & $\mathrm{Bg}_{9}$ & $\mathrm{~W}_{\mathrm{r}}{ }^{3}$ & $\mathrm{~W}_{1}^{0}{ }^{0}$ & $\lambda$ & $\mathrm{CI}$ \\
\hline $\mathrm{B}_{2}$ & 1 & 4 & 4 & $1 / 2$ & 12. & $2+$ & 1.414 & 0.188 & 6.307 & \\
\hline+ & 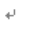 & & & & & & + & + & + & \\
\hline$B_{3}$ & $1 / 4$ & 1 & 1 & $1 / 5$ & $1 / 5$. & $1+$ & 0.464 & 0.062 & 6.048 & \\
\hline+ & + & & & & & & + & + & + & \\
\hline $\mathrm{B}_{4}$ & $1 / 4$ & 1 & $d$ & $1 / 4$ & $1 / 5$ & 1 & $0.589+$ & 0.078 & 6.346 & \\
\hline$\omega$ & + & & & & & & 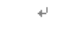 & + & $\checkmark$ & $0.075 \leq 0.1$ \\
\hline $\mathrm{B}_{5}$ & 2 & 5 & 4 & 1 & 2 & 3 & 2.493 & 0.332 & 6.256 & \\
\hline 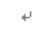 & + & & & & & & + & + & $\checkmark$ & \\
\hline$B_{8}$ & 2 & 5 & 3 & $1 / 2$ & 1 & 5 & 2.054 & 0.273 & 6.205 & \\
\hline+ & + & & & & & & 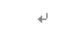 & + & $\checkmark$ & \\
\hline $\mathrm{Bg}_{9}$ & $1 / 2$ & 1 & $1 / 2$ & $1 / 3$ & $1 / 5$ & $1+$ & 0.505 & 0.067 & 6.377 & \\
\hline 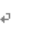 & 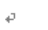 & & & & & & 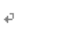 & 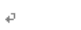 & . & P \\
\hline
\end{tabular}


Table 1-9

\begin{tabular}{|c|c|c|c|c|c|c|c|c|}
\hline \multicolumn{5}{|c|}{ Expert $D^{\prime}$ criteria matrix $\mathrm{A}=\left(\mathrm{z} \mathrm{z}_{1}\right)^{2}$} & \multicolumn{2}{|c|}{ A eiggen vector We } & \multicolumn{2}{|c|}{ A consistency check } \\
\hline $\mathrm{A}_{3} P$ & $B_{2}$ & $\mathrm{~B}_{7}$ & $\mathrm{~B}_{8}$ & $B_{10}$ & $\mathrm{w}_{\mathrm{I}^{2}}$ & $\mathrm{~W}_{\mathrm{T}}^{0} \mathrm{~F}$ & $\lambda$ & $\mathrm{CI}$ \\
\hline $\mathrm{B}_{2}$ & 1 & 5 & $1 / 2$ & 4 & 1.778 & 0.364 & 4.217 & \\
\hline $\mathrm{B}_{7}$ & $1 / 5$ & 1 & $1 / 3$ & 1 & $0.508+$ & 0.104 & 4.110 & \\
\hline 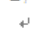 & 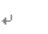 & & & & 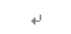 & + & + & $0002<0$ \\
\hline $\mathrm{B}_{8}$ & 2 & 3 & 1 & $3+$ & $2.060+$ & $0.422+$ & $4.246+$ & $0.082 \leq 0.1+$ \\
\hline 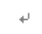 & 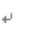 & & & & 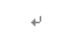 & 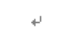 & + & \\
\hline$B_{10}$ & $1 / 4$ & 1 & $1 / 3$ & 1 & 0.537 & 0.110 & 4.052 & \\
\hline
\end{tabular}

(2): Comprehensive judgment

Some experts perform satisfaction assessment to obtain initial result according to index reference and their understanding of the evaluated objects and perform fuzzy comprehensive assessment to clarify whether production capacity satisfy product requirements. It is a method that evaluation system is comprehensively assessed by fuzzy set theory.

The evaluation factor sets are first order index set (A) and second order index set (B), assessment set $(\mathrm{E})$.

$\mathrm{E}=(\mathrm{e}$ I) $\Delta=$ (Extremely good, good, slightly good, ordinary, poor)

$\Delta=(0.9 、 0.7 、 0.5 、 0.3 、 0.1)$

Since different members give different assessments, the results can only be described by possibility of conducting e I assessment of question, with basic assessment format shown as Table 110:.

Table 1-10

\begin{tabular}{|c|c|c|c|c|c|c|c|c|}
\hline \multirow[b]{2}{*}{$\begin{array}{l}\text { First order } \\
\text { index }\end{array}$} & \multirow[b]{2}{*}{$\begin{array}{l}\text { Index } \\
\text { weight }\end{array}$} & \multirow[b]{2}{*}{$\begin{array}{l}\text { First order } \\
\text { index }\end{array}$} & \multirow[b]{2}{*}{$\begin{array}{l}\text { Index } \\
\text { weight }\end{array}$} & \multicolumn{5}{|c|}{ Satisfaction } \\
\hline & & & & $\begin{array}{c}\text { Extre } \\
\text { mely } \\
\text { good } \\
0.9\end{array}$ & $\begin{array}{c}\text { Good } \\
0.7\end{array}$ & $\begin{array}{c}\text { Slightly } \\
\text { good } \\
0.5\end{array}$ & $\begin{array}{l}\text { Ordina } \\
\text { ry } 0.3\end{array}$ & $\begin{array}{l}\text { Poor } \\
0.1\end{array}$ \\
\hline$A$ & $W_{A_{i}}$ & $\mathrm{~B}_{1} \mathrm{P}^{\mathrm{y}} \mathrm{C}$ & $W_{b^{4}}^{f^{2}}+$ & $m^{\rho}+$ & $r^{p} p^{2}$ & $\gamma_{B}{ }^{p}$ & $n^{f}$ & $\pi \mathbb{N}^{\rho^{+}}$ \\
\hline
\end{tabular}

$\sum \gamma=1$

By fuzzy evaluation method, assessment vector is indicated by fuzzy matrix obtained multiplying them, so single-factor evaluation vector of $\mathrm{Aj}$ is: By fuzzy evaluation method, assessment vector is indicated by fuzzy matrix. The fuzzy matrix is obtained by the product of them.

$$
\begin{gathered}
\mathrm{Sj}=\operatorname{Wbi}(\mathrm{j}) \mathrm{R}(\mathrm{j})=(\mathrm{Wbi}(\mathrm{j})) * \gamma \mathrm{il}=\mathrm{Sl}(\mathrm{j}) \quad \mathrm{l}=1-3 \\
\mathrm{R}=(\mathrm{Sjl}) \quad \mathrm{j}=1-10 \quad \mathrm{l}=1-3
\end{gathered}
$$

Comprehensive evaluation vector of product is: $\mathrm{S}=\mathrm{WAR}=（ \mathrm{WAj} ） \quad(\mathrm{Sjl})=(\mathrm{SI}) \quad \mathrm{l}=1-3$

Comprehensive assessment result of product use comprehensive satisfaction $\alpha$ is indicated as: $\alpha=\mathrm{SA}$

\section{Evaluation Process and Result}

After establishing evaluation system and determining system evaluation method, 10 persons in administration, technology and production line inside and outside the company were arranged to make satisfactory assessment by form-filling, with initial evaluation result shown as Table 1-11: 
Table 1-11

\begin{tabular}{|c|c|c|c|c|c|c|}
\hline \multirow{3}{*}{ 焉 } & \multirow{3}{*}{ 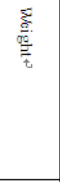 } & \multirow{3}{*}{ 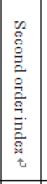 } & \multirow{3}{*}{ 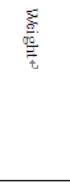 } & Product $\mathrm{A}$ & \multirow{2}{*}{$\begin{array}{l}\text { Product B } \\
\text { Satisfaction }\end{array}$} & \multirow{2}{*}{$\begin{array}{l}\text { Product C } \\
\text { Satisfaction }\end{array}$} \\
\hline & & & & Satisfaction & & \\
\hline & & & & $\begin{array}{l}\text { Extremely good, good, } \\
\text { slightly good, ordinary, } \\
\text { poor } \\
0.90 .70 .5 \quad 0.30 .1\end{array}$ & $\begin{array}{l}\text { Extremely good, good, } \\
\text { slightly good, ordinary, } \\
\text { poor } \\
0.90 .70 .50 .30 .1\end{array}$ & $\begin{array}{l}\text { Extremely good, good, } \\
\text { slightly good, ordinary, } \\
\text { poor } \\
0.90 .70 .5 \quad 0.30 .1\end{array}$ \\
\hline $\mathrm{A}_{1^{*}}$ & 0.226 & \begin{tabular}{|l|}
$B_{1}$ \\
$B_{3^{4}}$ \\
$B_{6^{4}}$ \\
\end{tabular} & $\begin{array}{l}0.1090 \\
5820.30 \\
9 \\
\end{array}$ & $\begin{array}{l}0.10 .50 .4 \\
0.50 .30 .1 \\
0.60 .10 .3\end{array}$ & $\begin{array}{ll}0.30 .60 .1 \\
0.50 .4 & 0.1 \\
0.70 .2 & 0.1 \\
\end{array}$ & $\begin{array}{l}0.10 .50 .30 .2 \\
0.30 .40 .3 \\
0.40 .10 .5 \\
\end{array}$ \\
\hline $\mathrm{A}_{2+}^{+}$ & 0.673 & \begin{tabular}{l|}
$\mathrm{B}_{2}$ \\
$\mathrm{~B}_{34}$ \\
$\mathrm{~B}_{4}$ \\
$\mathrm{~B}_{5}$ \\
$\mathrm{~B}_{34}$ \\
$\mathrm{~B}_{9}$
\end{tabular} & $\begin{array}{l}0.1880 \\
0620.07 \\
80.3320 \\
.2730 .0 \\
67\end{array}$ & $\begin{array}{l}0.60 .30 .1 \\
0.40 .50 .1 \\
0.50 .30 .2 \\
0.50 .30 .1 \\
0.70 .2 \\
0.70 .20 .1\end{array}$ & $\begin{array}{l}0.70 .3 \\
0.60 .30 .1 \\
0.50 .5 \\
0.50 .4 \\
0.90 .1 \\
0.80 .1\end{array}$ & $\begin{array}{l}0.40 .20 .4 \\
0.30 .40 .3 \\
0.50 .30 .2 \\
0.30 .40 .3 \\
0.50 .20 .1 \\
0.70 .2 \quad 0.2 \\
\end{array}$ \\
\hline$A_{3}+$ & 0.101 & \begin{tabular}{l|}
$\mathrm{B}_{2}$ \\
$\mathrm{~B}_{7}$ \\
$\mathrm{~B}_{8}$ \\
$\mathrm{~B}_{1}$ \\
$0^{4}$
\end{tabular} & $\begin{array}{l}0.3640 \\
1040.42 \\
20.110\end{array}$ & $\begin{array}{l}0.60 .30 .1 \\
0.60 .20 .1 \\
0.70 .2 \quad 0.1 \\
0.50 .30 .2\end{array}$ & $\begin{array}{l}0.70 .3 \\
0.60 .30 .1 \\
0.90 .1 \\
0.50 .40 .1\end{array}$ & $\begin{array}{l}0.40 .20 .4 \\
0.30 .40 .3 \\
0.50 .20 .1 \\
0.30 .30 .4\end{array}$ \\
\hline
\end{tabular}

According to initial evaluation result, obtain the evaluation result of first order index $\mathrm{Aj}$ (represented by evaluation vector $\mathrm{Sj}$ ), shown as Table 1-12: According to the initial evaluation results, the evaluation result of first order index $\mathrm{Aj}$ is reached.

Table 1-12

\begin{tabular}{|c|c|c|c|}
\hline $\mathrm{A}_{\mathrm{s}}-\mathrm{S}_{\mathrm{s}} \mathrm{P}$ & Product A & Product $B$ & Product $\mathrm{C}$ \\
\hline $\mathrm{S}_{1}$ & $0.487,0.26,0.195,0.058$, of & $0.54,0.36,0.011,0.089,04$ & $0.309,0.318,0.362,0.022,0.4$ \\
\hline $\mathrm{S}_{2}$ & $0.581,0.278,0.081,0.050$, of & $0.673,0.281,0.013,0.033,0 f$ & $0.416,0.287,0.236,0.061,0.4$ \\
\hline $\mathrm{S}_{3}$ & $0.631,0.247,0.069,0.053$, of & $0.752,0.227,0.021,0,0$ & $0.421,0.232,0.263,0.084,0.7$ \\
\hline
\end{tabular}

Obtain comprehensive evaluation vectors of products $A, B, C$ respectively: comprehensive evaluation vectors of product $\mathrm{A}, \mathrm{B}$ and $\mathrm{C}$ are as follows:

$\mathrm{SA}=(0.565,0.271,0.106,0.064,0)$

$\mathrm{SB}=(0.651,0.293,0.013,0.042,0)$

$\mathrm{SC}=(0.392,0.288,0.267,0.055,0)$

Further obtain the evaluation results shown by comprehensive satisfaction: And then it is reached that evaluation results can be indicated by comprehensive satisfaction as shown bellow:

$\alpha \mathrm{A}=0.770$

$$
\begin{aligned}
& \alpha \mathrm{B}=0.810 \\
& \alpha \mathrm{C}=0.704
\end{aligned}
$$

Comprehensive evaluation results indicate the company has the capacity of machining product $\mathrm{B}$ well. Comprehensive evaluation results indicate that the company has a better capacity to produce product B.

\section{Conclusion}

As a strongly practical subject, system engineering shall be integrated closely with practice. By the very philosophy and research method of going from theory to practice and then generalizing theory from practice, this paper analyzes main factors contributing to the company's production capacity by structure modeling, hierarchy analysis, fuzzy evaluation, etc., solving the decision-making of foreign order for the good reference for similar companies. The system engineering is a very practical subject and needs closely integrated with practice. This paper absolutely follows the philosophy and 
research method of going from theory to practice and then generalizing theory from practice. Take XX Precise Machining Company as empirical research object, this paper analyzed the key impact factors of company's production capacity and solved problems of production decision-making in foreign orders by system engineering methods as structural modeling, level analysis, and fuzzy evaluation. This case has important reference value for the similar companies.

\section{References}

[1] Yingluo Wang.System engineering [M]. Beijing: Mechanical Engineering press,2016

[2] Yongbo Lu. System engineering [M]. Beijing: Beijing Jiaotong University press,2006 